\title{
A Simple Expression for the Center of Mass of a System of Particles in a Two-Dimensional Space with Constant Positive Curvature
}

\author{
${ }^{1}$ Pedro P. Ortega Palencia, ${ }^{2}$ Ruben Dario Ortiz and ${ }^{3} \mathrm{~J}$. Guadalupe Reyes Victoria \\ ${ }^{1}$ Ecuaciones Diferenciales Research Group, Department of Mathematics, Campus San Pablo, \\ ${ }^{2}$ ONDAS Research Group, Department of Mathematics, Campus San Pablo, Universidad de Cartagena, \\ Avenida del Consulado Cra 50\#24-120, 130014 Cartagena de Indias, Colombia \\ ${ }^{3}$ Department of Mathematics, Universidad Autónoma Metropolitana, Avenida San Rafael Atlizco 186, \\ Colonia Vicentina, 09340 Iztalapa, CDMX, Mexico \\ portegap@unicartagena.edu.co
}

\begin{abstract}
In this research is given a simple expression for the center of mass of a system of material points in a two-dimensional surface of Gaussian constant positive curvature. Using basic techniques of geometry, an expression in intrinsic coordinates is obtained and it is showed how this extend the definition for the Euclidean case. The argument is constructive and also serves for defining center of mass of a system of particles on the one-dimensional Sphere $s_{\mathrm{R}}^{1}$.
\end{abstract}

Key words: Center of masses, conformal metric, spherical rule of the lever, geodesic, Euclidean case, one-dimensional sphere

\section{INTRODUCTION}

Center of mass (center of gravity or centroid) is a fundamental concept and its geometrical and mechanics properties are very important in the comprehension of a great variety of physical problems. Their definition for Euclidean spaces is elemental, nevertheless a definition for curved spaces is few frequent. Galperin (1993) makes an extensive explanation showing the possibilities of construction this concept in more general spaces and it is signalized the difficulties to defined in spaces of non zero curvature because this lack of linear structure. While it is true that the researcher synthesizes the basic properties of the center of mass in his approach appear some ones lacking of physical meaning such as the non conservation of total mass of system, in normal conditions or the presence of infinities velocities. Diacu et al. (2012) makes mention about of the difficulty for defining of center of mass in curved spaces. He provides a class of orbits in the curved n-body problem for which apparently "no point that could play the role of the center of mass is fixed or moves uniformly along a geodesic". This proves that the equations of motion lack center-of-mass and linear-momentum integrals. But nevertheless, it is not provide a way to calculate or determinate this element. Applications in fields like Chemistry by Berrio-Guzman et al. (2015). By Aarseth (2003), it can found gravitational $n$-body simulations. The gravitational million-body problem has a multidisciplinary approach to star cluster dynamics (Heggie and Hut, 2003). Barnes and Hut (1986) was obtained a hierarchical $O(N \log N)$ forcecalculation algorithm. Initial conditions for star clusters was taken for the dynamics of the $\mathrm{n}$ body problem (Kroupa, 2008). A good book for an introduction to celestial mechanics (Moulton, 1970). An algorithm for finding best matches in logarithmic expected time it is useful for codes in n-body problems (Friedman et al., 1977). Steinbach and Brooks (1994) obtain new sphericalcutoff methods for long-range forces in macromolecular simulation in this case the potential is similar to the potential for n-body problems. Multifocus image fusion using improved dual tree complex wavelet transform and discrete optimization method, this method of dual tree is useful for simulation in n-body problems (Srilatha, 2014). Location of collinear equilibrium points in the generalized photogravitational elliptic restricted three body problem is a difficult problem (Kumar and Ishwar, 2011). Artificial satellites, center of mass and the three body problem are related. For artificial satellites (Hillier and Balyan, 2019; Si Mohammed et al., 2007; Cooksley et al., 2007;

Corresponding Author: Pedro P. Ortega Palencia, Ecuaciones Diferenciales Research Group, Deparment of Mathematics, Campus San Pablo, Universidad de Cartagena, Avenida del consulado Cra 50 \# 24-120, 130014Cartagena de Indias, Colombia, portegap@unicartagena.edu.co 
Adediji et al., 2007; Ahmed et al., 2010; Emetere et al., 2016; Singh, 2017; Anand, 2017; Buliali et al., 2017; Phonphan, 2017; Basha and Vijayakumar, 2018; Nathan, 2017; Parthasarathy, 2017; Kaur and Singh, 2017; Rajab et al., 2018; Ramakrishnan, 2018; Nathan, 2018; Manivannan, 2018; Ik-Soo and Myung-Jin Bae, 2019; Sergey et al., 2019; Ashraf et al., 2019).

Garcia-Naranjo et al. (2016) in which they refer to a center of masses in a curved space, in a problem of celestial mechanics but they do not give a formula or a procedure to calculate it. Diacu (2012) study the n-body problem in spaces of constant curvature. Ortega et al. (2019) find the hyperbolic center of mass for a system of particles on the Poincare upper half-plane. Diacu et al. (2018) investigate the stability of fixed points and associated relative equilibria of the 3-body problem on $\mathrm{S}^{1}$ and $S^{2}$. Shchepetilov (2006) is a book of mechanics on curved spaces.

In this research, the problem of gives a mathematical expression for computing the center of mass of a system of $\mathrm{n}$ particles sited on the two-dimensional sphere with Ratio $\mathrm{R}, \mathrm{S}_{\mathrm{R}}^{2}$ is considered. Through stereographic projection of $\mathrm{S}_{\mathrm{R}}^{2}$ on the extend Complex plane $\hat{\mathrm{C}}$, endowed with the conformal metric (Perez-Chavela and Reyes-Victoria, 2012):

$$
\mathrm{ds}^{2}=\frac{4 \mathrm{R}^{4} \mathrm{dwd} \overline{\mathrm{w}}}{\left(\mathrm{R}^{2}+|\mathrm{w}|^{2}\right)^{2^{\prime}}}
$$

Both, $\hat{C}$ with the metric (Eq. 1) and $s_{\mathrm{R}}^{2}$ with the Euclidean metric have the same Gaussian curvature $\mathrm{K}=$ $1 / R^{2}$ and for the Minding's Theorem belong to the isometric differentiable class (Do Carmo, 1976; Dubrovin et al., 1984; Perez-Chavela and Reyes-Victoria, 2012) is proved the equivalence of the n-body problem for both models, the one on the sphere $S_{R}^{2}$ with the Euclidean metric of ambient space $\mathrm{R}^{3}$ and the other on the extend Complex plane $\hat{\mathrm{C}}$ with the metric (Eq. 1). Following the basic methods of the geometry, we obtain, here, the expression for the center of mass for a system of $n$ particles sited in the sphere $S_{\mathrm{R}}^{2}$ of arbitrary Radio $\mathrm{R}$.

This study is organized as follow: in section 1 are introduced some concepts relative to center of mass in the euclidean spaces. In section 2 , are remembered some properties of stereographical projection and it is proceeded to deduce the expression for the center of mass, for two particles on the sphere from the "spherical rule of the lever" (Galperin, 1993) extended to surface of $S_{R}^{2}$, this case can be reduced to the Sphere $S_{R}^{1}$, under a suitable two rotations and using the invariance of the relative position of center of mass under isometrics of sphere (Galperin, 1993). Once obtained the expression for the center of mass for two particles in $S_{R}^{1}$, it can be extended naturally to a system of $n$ particles in $S_{R}^{1}$ and the same way, to a system of particles in $\mathrm{S}_{\mathrm{R}}^{2}$. The expression obtained, here, satisfies the five axioms for the "Axiomatic Centroid" established by Galperin (1993).

\section{MATERIALS AND METHODS}

One-dimensional Euclidean case: Let consider two particles with positive masses sited in the real line at the points $x_{1}$ and $x_{2}$. The center of mass of system is defined be the point $x_{c}$ :

$$
\mathrm{x}_{\mathrm{c}}=\frac{\mathrm{m}_{1} \mathrm{x}_{1}+\mathrm{m}_{2} \mathrm{x}_{2}}{\mathrm{~m}_{1}+\mathrm{m}_{2}}
$$

A direct calculation shows that $m_{1}\left|x_{c}-x_{1}\right|=m_{2}\left|x_{c}-x_{2}\right|$ (Euclidean rule of the lever). It is easy to prove that $x_{c}$ is the unique point in the segment (geodesic) joining $x_{1}$ and $\mathrm{x}_{2}$ with this property. This definition can be extend to more dimensions in Euclidean spaces. This definition can not be extended to spaces in general because is possible that in such spaces is not defined a linear structure. But with the "rule of the lever" in mine is possible carries this definition to Riemannian surfaces as we shall see later.

In Fig. 1 showed that: $s_{1}=\theta_{1} R, s_{2}=\theta_{2} R \tan \theta_{1}=d_{1} / R$ and $\tan \theta_{2}=d_{2} / R$. Moreover, when $R \rightarrow \infty, \theta_{1}, \theta_{2} \rightarrow 0$ and $\mathrm{s}_{1} \rightarrow \mathrm{d}_{1}$ y $_{2} \rightarrow \mathrm{d}_{2}$ then,

$$
1=\lim _{\mathrm{R} \rightarrow \infty} \frac{\tan \theta_{1}}{\theta_{1}}=\lim _{\mathrm{R} \rightarrow \infty} \frac{\mathrm{d}_{1} / \mathrm{R}}{\mathrm{S}_{1} / \mathrm{R}}=\lim _{\mathrm{R} \rightarrow \infty} \frac{\mathrm{d}_{1}}{\mathrm{~S}_{1}}
$$

Hence, $\mathrm{s}_{1} \rightarrow \mathrm{d}_{1}$ and in similar way $\mathrm{s}_{2} \rightarrow \mathrm{d}_{2}$.

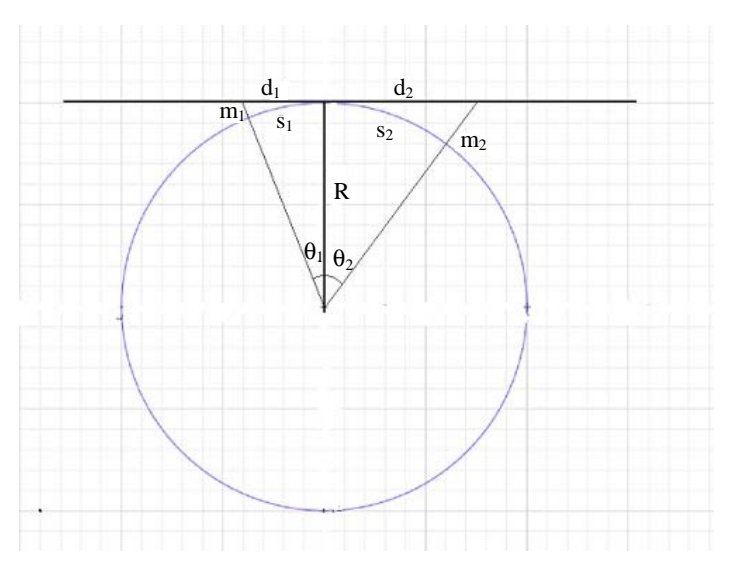

Fig. 1: Center of masses in the circumference and the real line 


\section{RESULTS AND DISCUSSION}

Center of masses in a two-dimensional spherical space Some observations about the stereographic projection: Let $\mathrm{P}: \mathrm{S}_{\mathrm{R}}^{2} \rightarrow \widehat{\mathrm{C}}$ the stereographic projection, then for $(x, y, z) \in S_{R}^{2}$, we have $P(x, y, z)=w=u+i v$ where $u=R x / R-z$ and $\mathrm{v}=\mathrm{Ry} / \mathrm{R}-\mathrm{z}$ and $\mathrm{P}(0,0, \mathrm{R})=\infty$ and moreover the inverse projection is $\mathrm{P}^{-1}: \widehat{\mathrm{C}} \rightarrow \mathrm{S}_{\mathrm{R}}^{2}$ and:

$$
\mathrm{P}^{-1}(\mathrm{u}+\mathrm{iv})=\frac{2 \mathrm{R}^{2} \mathrm{u}}{\mathrm{u}^{2}+\mathrm{v}^{2}+\mathrm{R}^{2}}, \frac{2 \mathrm{R}^{2} \mathrm{v}}{\mathrm{u}^{2}+\mathrm{v}^{2}+\mathrm{R}^{2}}, \frac{\mathrm{R}\left(\mathrm{u}^{2}+\mathrm{v}^{2}-\mathrm{R}^{2}\right)}{\mathrm{u}^{2}+\mathrm{v}^{2}+\mathrm{R}^{2}}
$$

$P$ leaves invariant the set $\left\{(x, y, z) \in S_{R}^{2}: z=0\right\}$ and it is the same set and will called equator. For analogy, the set $\{\mathrm{W} \in \mathrm{C}:|\mathrm{W}|<\mathrm{R}\}$ will called South Hemisphere and the set $\{\mathrm{W} \in \mathrm{C}: \mid \mathrm{W}>\mathrm{R}\}$ will called North Hemisphere, $\mathrm{W}=0$ is called South Pole and $\mathrm{w}=\infty$ will be called North Pole. $\mathrm{P}^{-1}$ transform lines through the origin in meridians and the circles with center in origin, $\{\mathrm{W} \in \mathrm{C}:|\mathrm{w}|<$ const. $\}$ in parallels. Finally, for two complex numbers $\mathrm{w}_{1}, \mathrm{w}_{2}$, if $\left|\mathrm{w}_{1}\right|\left|\mathrm{w}_{2}\right|=\mathrm{R}^{2}$, then each point is sited in two parallels, the same radio and symmetric respect to Equator.

If consider the stereographic projection of the onedimensional sphere $S_{\mathrm{R}}^{2}$ on the real line, then the above equation is reduced to:

$\mathrm{P}(\mathrm{x}, \mathrm{y})=\mathrm{u}$ where $\mathrm{u}=\mathrm{Rx} / \mathrm{R}-\mathrm{y}$ and $\mathrm{P}(0, \mathrm{R})=\infty$ and moreover the inverse projection is:

$$
P^{-1}(u)=\left(\frac{2 R^{2} u}{u^{2}+R^{2}}, \frac{R\left(u^{2}-R^{2}\right)}{u^{2}+R^{2}}\right)
$$

In this last case, the length of arc from the South Pole $\mathrm{P}_{\mathrm{s}}$ to arbitrary point $(\mathrm{x}, \mathrm{y})$ is:

$$
\mathrm{s}=\left.\right|_{0} ^{\mathrm{u}} \frac{2 \mathrm{R}^{2} \mathrm{dt}}{\mathrm{t}^{2}+\mathrm{R}^{2}}=2 \mathrm{R} \arctan \left(\frac{\mathrm{u}}{\mathrm{R}}\right)
$$

More general, the length of arc $\mathrm{s}$ from the point $\mathrm{Q}_{1}\left(\mathrm{x}_{1}\right.$, $\left.\mathrm{y}_{1}\right)$ to $\mathrm{Q}_{2}\left(\mathrm{x}_{2}, \mathrm{y}_{2}\right)$, if their stereographical projections are $\mathrm{u}_{1}$ and $\mathrm{u}_{2}$ is:

$$
\mathrm{s}=2 \mathrm{R}\left(\arctan \left(\frac{\mathrm{u}_{2}}{\mathrm{R}}\right)-\arctan \left(\frac{\mathrm{u}_{1}}{\mathrm{R}}\right)\right)
$$

Consider now two masses $\mathrm{m}_{1}, \mathrm{~m}_{2}$ sited in the points $Q_{1}, Q_{2}$, respectively and let $Q_{c}\left(x_{c}, y_{c}\right)$ the coordinates of center of mass and $s_{1}$ the length of arc from $Q_{1}$ to $Q_{2}$ and $s_{2}$ the length of arc from $Q_{c}$ to $Q_{2}$. Then, from the relation (spherical rule of the lever) $\mathrm{m}_{1} \mathrm{~s}_{1}=\mathrm{m}_{2} \mathrm{~s}_{2}$ it follows:

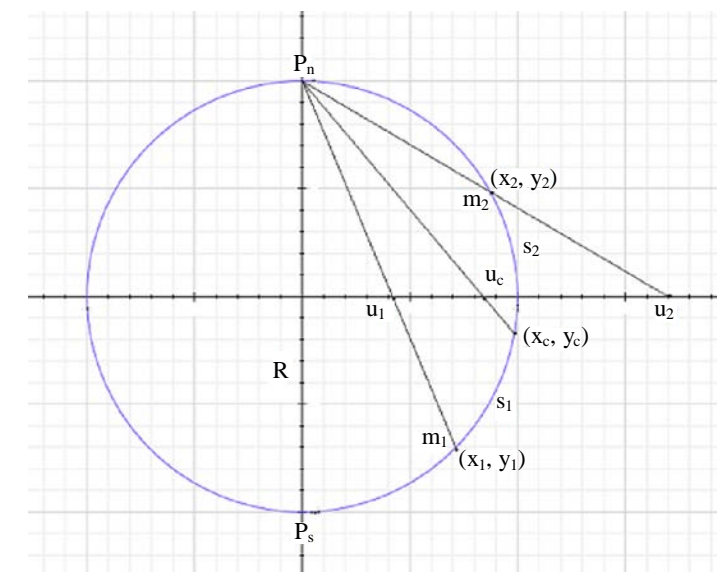

Fig. 2: Center of mass on the one-dimensional Sphere $S_{R}^{2}$

$$
\begin{aligned}
& 2 \mathrm{Rm}_{1}\left(\arctan \left(\frac{\mathrm{u}_{\mathrm{c}}}{\mathrm{R}}\right)-\arctan \left(\frac{\mathrm{u}_{1}}{\mathrm{R}}\right)\right) \\
& =2 \mathrm{Rm}_{2}\left(\arctan \left(\frac{\mathrm{u}_{2}}{\mathrm{R}}\right)-\arctan \left(\frac{\mathrm{u}_{\mathrm{c}}}{\mathrm{R}}\right)\right)
\end{aligned}
$$

Therefore:

$\operatorname{Arctan}\left(\frac{\mathrm{u}_{\mathrm{c}}}{\mathrm{R}}\right)=\frac{1}{\mathrm{~m}_{1}+\mathrm{m}_{2}}\left(\mathrm{~m}_{1} \arctan \left(\frac{\mathrm{u}_{1}}{\mathrm{R}}\right)+\mathrm{m}_{2} \arctan \left(\frac{\mathrm{u}_{2}}{\mathrm{R}}\right)\right)$

Figure 2 illustrates the situation. This concept can be extended a $n$ particles with masses $m_{1}, m_{2}, \ldots, m_{n}$ sited in the points $\left(\mathrm{x}_{1}, \mathrm{y}_{1}\right),\left(\mathrm{x}_{3} \mathrm{y}_{2}\right), \ldots,\left(\mathrm{x}_{\mathrm{n}} \mathrm{y}\right)$ on $\mathrm{s}_{\mathrm{R}}^{2}$ and with stereographical projections $\mathrm{u}_{1}, \mathrm{u}_{2}, \ldots, \mathrm{u}_{\mathrm{n}}$ in the real line the following way:

$$
\operatorname{Arctan}\left(\frac{\mathrm{u}_{\mathrm{c}}}{\mathrm{R}}\right)=\frac{1}{\mathrm{~m}} \sum_{\mathrm{k}=1}^{\mathrm{n}} \mathrm{m}_{\mathrm{k}} \arctan \left(\frac{\mathrm{u}_{\mathrm{k}}}{\mathrm{R}}\right)
$$

where, $\mathrm{m}=\sum_{\mathrm{k}=1}^{\mathrm{n}} \mathrm{m}_{\mathrm{k}}$.

Center of masses for a system of two particles in $\mathbf{S}_{{ }_{\mathrm{R}}}$ : Now, we extend the "rule of the lever" to context more general: let a Riemannian surface $\mathrm{T}$ and two particles with masses $m_{1}, m_{2}$ sited in the points $s_{1}, s_{2} \in T$, respectively, then the center of mass is defined the point $s_{c}$ in the geodesic joining $s_{1}$ to $s_{2}$ such that is verified the following relation:

$$
\mathrm{m}_{1} \mathrm{~d}\left(\mathrm{~s}_{1}, \mathrm{~s}_{\mathrm{c}}\right)=\mathrm{m}_{2} \mathrm{~d}\left(\mathrm{~s}_{2}, \mathrm{~s}_{\mathrm{c}}\right)
$$

where, $d$ is the metric in T. For the case of $S_{R}^{2}$, geodesics are great circles and distances are measure take the shorter arc.

Let $m_{1}, m_{2}, \ldots, m_{n}, n$ masses sited, respectively in the points $\left(x_{1}, y_{1}, z_{1}\right),\left(x_{2}, y_{2}, z_{2}\right), \ldots,\left(x_{n}, y_{n}, z_{n}\right)$ in $S_{R}^{2}$ with 
stereographical projections $\mathrm{w}_{1}, \mathrm{w}_{2}, \ldots, \mathrm{w}_{\mathrm{n}}$ in the extend complex plane and let $\mathrm{w}_{\mathrm{c}}$ their center of mass, then it is satisfy the next relation:

$$
\operatorname{Arctan}\left(\frac{\mathrm{w}_{\mathrm{c}}}{\mathrm{R}}\right)=\frac{1}{\mathrm{~m}} \sum_{\mathrm{k}=1}^{\mathrm{n}} \mathrm{m}_{\mathrm{k}} \arctan \left(\frac{\mathrm{w}_{\mathrm{k}}}{\mathrm{R}}\right)
$$

where, $\mathrm{m}=\sum_{\mathrm{k}=1}^{\mathrm{n}} \mathrm{m}_{\mathrm{k}}$. Note that in 5 , if multiplied by $\mathrm{R}$ in both sides and we take the limit when $\mathrm{R} \rightarrow \infty$ then result:

$$
\mathrm{w}_{\mathrm{c}}=\frac{1}{\mathrm{~m}} \sum_{\mathrm{k}=1}^{\mathrm{n}} \mathrm{m}_{\mathrm{k}} \mathrm{w}_{\mathrm{k}}
$$

And this correspond to the equation for the center of mass in the Euclidean complex plane, that is the complex plane (or $\mathrm{R}^{2}$ ), with Euclidean metric and zero curvature.

\section{CONCLUSION}

There is a formula to calculate centers of mass in curved spaces that extends this concept of Euclidean spaces (of null curvature).

\section{ACKNOWLEDGEMENT}

The researchers express their deep gratitude to Universidad de Cartagena. Cartagena de Indias, Colombia for financial support [Grant Numbers 066-2013, 117-2017] and the Universidad Autónoma Metropolitana. Iztapalapa, Ciudad de México, México.

\section{STATEMENT OF SIGNIFICANCE}

In this research we present for the first time a formula to calculate centers of mass in curved spaces.

\section{REFERENCES}

Aarseth, S.J., 2003. Gravitational N-Body Simulations: Tools and Algorithms. Cambridge University Press, Cambridge, UK., ISBN:9780521432726, Pages: 413. Adediji, A.T., M.O. Ajewole, M.T. Babalola and R.A. Adenodi, 2007. A study ofaudio satellite communication system analysis in Akure South West, Nigeria. J. Eng. Applied Sciences, 2: 467-471.

Ahmed, O.D., E.E. Omizegba and M.I. Onogu, 2010. Orbital mechanism and satellite orbital location. J. Eng. Appl. Sci., 5: 276-281.

Anand, N., 2017. Framelet transform based satellite image enhancement. J. Eng. Appl. Sci., 12: 4169-4171.
Ashraf, S.A., Q.B. Al-Abudi and M.S. Mahdi, 2019. Distribution of land surface temperatures from satellite images for Al-hammar marshes in Iraq. J. Eng. Appl. Sci., 14: 3651-3658.

Barnes, J. and P. Hut, 1986. A hierarchical O (N $\log \mathrm{N})$ force-calculation algorithm. Nature, 324: 446-449.

Basha, S.A. and V. Vijayakumar, 2018. Wavelet transform based satellite image enhancement. J. Eng. Applied Sci., 13: 854-856.

Berrio-Guzman, S.J., C. de Indias, A.M. Marin-Ramirez and R.D. Ortiz-Ortiz, 2015. Gaussian curvature in a p-orbital, hydrogen-like atoms. Adv. Stud. Theor. Phys., 9: 281-285.

Buliali, J.L., C. Fatichah, D. Herumurti, D. Fenomena, H. Widyastuti and M. Wallace, 2017. Vehicle detection on images from satellite using oriented fast and rotated brief. J. Eng. Appl. Sci., 12: 4500-4503.

Cooksley, J.R., L.H. Abderrahmane, M. Benyettou, M.N. Sweeting and P. Garner, 2007. Generic S-band radio frequency system for earth observation leo satellite. J. Eng. Applied Sciences, 2: 286-289.

Diacu, F., 2012. The non-existence of centre-of-mass and linear-momentum integrals in the curved N-body problem. Libertas Math, 32: 25-37.

Diacu, F., E. Perez-Chavela and M. Santoprete, 2012. The N-body problem in spaces of constant curvature, Part I: Relative equilibria. J. Nonlinear Sci., 22: 247-266.

Diacu, F., J.M. Sanchez-Cerritos and S. Zhu, 2018. Stability of fixed points and associated relative equilibria of the 3-body problem on $\mathrm{S}^{1}$ and $\mathrm{S}^{2}$. J. Dyn. Differ. Equations, 30: 209-225.

Do Carmo, M.P., 1976. Differential Geometry of Curves and Surfaces. Prentice Hall, New Jersey, USA., ISBN:9780132125895, Pages: 503.

Dubrovin, B., A. Fomenko and P. Novikov, 1984. Modern Geometry-Methods and Applications: Part I The Geometry of Surfaces, Transformation Groups and Fields. 2nd Edn., Springer, Berlin, Germany, ISBN:9780387908724, Pages: 464.

Emetere, M.E., M.L. Akinyemi and O. Akinojo, 2016. Effects of band superposition on the satellite imagery of aerosol optical depth over west Africa. J. Eng. Appl. Sci., 11: 17-22.

Freidman, J.H., J.L. Bentley and R.A. Finkel, 1977. An algorithm for finding best matches in logarithmic expected time. ACM Trans. Math. Software, 3: 209-226.

Galperin, G.A., 1993. A concept of the mass center of a system of material points in the constant curvature spaces. Commun. Math. Phys., 154: 63-84. 
Garcia-Naranjo, L.C., J.C. Marrero, E. Perez-Chavela and M. Rodriguez-Olmos, 2016. Classification and stability of relative equilibria for the two-body problem in the hyperbolic space of dimension. J. Differ. Equations, 260: 6375-6404.

Heggie, D. and P. Hut, 2003. The Gravitational Million-Body Problem: A Multidisciplinary Approach to Star Cluster Dynamics. Cambridge University Press, Cambridge, UK., ISBN:9780521774864, Pages: 357.

Hillier, C., and V. Balyan, 2019. Effect of space radiation on LEO nanosatellites. J. Eng. Appl. Sci., 14: 6843-6857.

Ik-Soo, A. and B. Myung-Jin, 2019. A study on the prevention of smombie's accidents by satellite information. J. Eng. Appl. Sci., 14: 945-950.

Kaur, S. and H. Singh, 2017. Performance enhancement of ultra dense inter-satellite optical wireless communication system incorporating polarization diversity. J. Eng. Appl. Sci., 12: 8802-8808.

Kroupa, P., 2008. Initial Conditions for Star Clusters. In: The Cambridge N-Body Lectures, Aarseth, S.J., C.A. Tout and R.A. Mardling (Eds.). Springer, Berlin, Germany, ISBN:978-1-4020-8430-0, pp: 181-259.

Kumar, S. and B. Ishwar, 2011. Location of collinear equilibrium points in the generalised photogravitational elliptic restricted three body problem. Intl. J. Eng. Sci. Technol., 3: 157-162.

Manivannan, J., 2018. Mobile satellite service expand opportunity for ocean observing and monitoring using AIS. J. Eng. Appl. Sci., 13: 9374-9379.

Moulton, F.R., 1970. An Introduction to Celestial Mechanics. Courier Corporation, North Chelmsford, Chelmsford, Massachusetts, USA.,.

Nathan, L.S., 2018. Expectation maximization based satellite image segmentation. J. Eng. Appl. Sci., 13: 9343-9345.

Nathan, S., 2017. K-Means algorithm based satellite image segmentation. J. Eng. Appl. Sci., 12: 7995-799.
Ortega, P., R.D. Ortiz and A.M. Marin, 2019. Hyperbolic center of mass for a system of particles on the Poincare upper half-plane. Res. J. Appl. Sci., 14: 49-53.

Parthasarathy, S., 2017. Review of image denoising methods using satellite images. J. Eng. Appl. Sci., 12: 7998-8000.

Perez-Chavela, E. and J.G. Reyes-Victoria, 2012. An intrinsic approach in the curved ?-body problem: The positive curvature case. Trans. Am. Math. Soc., 364: 3805-3827.

Phonphan, W., 2017. Using regression models to predict electrical conductivity of soil through ALOS PALSAR satellite. J. Eng. Appl. Sci., 12: 7276-7279.

Rajab, J.M., I.S. Abdulfattah and H.A. Mossa, 2018. Monitoring dust storms over Iraq using satellite images a case study. J. Eng. Appl. Sci., 13: 3663-3670.

Ramakrishnan, I.V.S., 2018. Ship position data analytics with vessel speed using satellite. J. Eng. Appl. Sci., 13: 8111-8113.

Sergey, L., M.A. Ivanushkin and S.S. Volgin, 2019. Advancement of design technologies for small remote earth's sensing satellites. J. Eng. Appl. Sci., 14: 2853-2857.

Shchepetilov, A.V., 2006. Calculus and Mechanics on Two-Point Homogenous Riemannian Spaces. Springer, Berlin, Germany, ISBN:9783540353867, Pages: 242.

Si Mohammed, A.M., M. Benyettou, A. Boudjemai, S. Chouraqui, Y. Hashida and M.N. Sweeting, 2007. Nadir stabilised system of the alsat-1 first algerian microsatellite in orbit. J. Eng. Appl. Sci., 2: 275-281.

Singh, R.D., 2017. Tetrolet transform based satellite image enhancement. J. Eng. Appl. Sci., 12: 3930-3932.

Srilatha, K., 2014. Multifocus image fusion using improved dual tree complex wavelet transform and discrete optimization method. J. Eng. Appl. Sci., 9: 414-421.

Steinbach, P.J. and B.R. Brooks, 1994. New spherical-cutoff methods for long-range forces in macromolecular simulation. J. Comput. Chem., 15: $667-683$. 